\title{
Acknowledgement to Reviewers of Membranes in 2016
}

\author{
Membranes Editorial Office \\ Published: 11 January 2017 \\ MDPI AG, St. Alban-Anlage 66, 4052 Basel, Switzerland; membranes@mdpi.com
}

The editors of Membranes would like to express their sincere gratitude to the following reviewers for assessing manuscripts in 2016.

We greatly appreciate the contribution of expert reviewers, which is crucial to the journal's editorial process. We aim to recognize reviewer contributions through several mechanisms, of which the annual publication of reviewer names is one. Reviewers receive a voucher entitling them to a discount on their next MDPI publication and can download a certificate of recognition directly from our submission system. Additionally, reviewers can sign up to the service Publons (https://publons.com) to receive recognition. Of course, in these intiatives we are careful not to compromise reviewer confidentiality. Many reviewers see their work as a voluntary and often unseen part of their role as researchers. We are grateful to the time reviewers donate to our journals and the contribution they make.

If you are interested in becoming a reviewer for Membranes, see the link at the bottom of the webpage http://www.mdpi.com/reviewers.

The following reviewed for Membranes in 2016:

Hawa, Hani A.E.

Ahmadiannamini, Pejman

Albo, Jonathan

Ochoa, Nelio Ariel

Arnusch, Christopher

Artyushkova, Kateryna

Baltus, Ruth E.

Bechelany, Mikhael

Bolotov, Leonid

Bormashenko, Edward

Bos, Martine P.

Brant, Jonathan A.

Bremond, Nicolas

Brohawn, Stephen G.

Buonomenna, Maria Giovanna

Carbone, Alessandra

Casado-Coterillo, Clara

Chang, Yong Keun

Cho, Namjoon

Christofides, Panagiotis D.

Chung, Tai-Shung

Cioffi, Raffaele

Clochard, Marie-Claude

Colavita, Paula E.

Cornell, B.
Coronas, Joaquín

Cranfield, Charles

Darvishmanesh, Siavash

David, Lamuel

De Quirós, A. Rodríguez-Bernaldo

Diban, Nazely

Sizemore, Ioana E. Pavel

Elimelech, Menachem

Fanucchi, Sylvia

Fatyeyeva, Kateryna

Ferrari, Maria-Chiara

Galeazzi, Roberta

Garcia-Ivars, Jorge

Glaubitz, Clemens

Grilli, Selene

$\mathrm{Gu}$, Yibei

Gupta, Shuchi

Hettiarachchi, Mala

Hirtz, Michael

Holzapfel, Sebastian

Hong, Liang

$\mathrm{Hu}$, Meng

Mendizábal, Raquel Ibáñez

Imbrogno, Alessandra

Irabien, Angel 
Javadi, Ali

Ji, Peng

Jin, Xinfang

Jørgensen, Mads K.

Kang, Dun-Yen

Kang, Sang Wook

Kim, Han-seung

Kim, Jaheon

Kimura, Katsuki

Koeppe II, Roger E.

Köper, Ingo

Kulozik, Ulrich

Lansman, Jeffry B.

Lee, Sunwoo

Lesage, Geoffroy

Li, Qian

Lin, Shihong

Lin, Yi-Li

Lisak, Grzegorz

Littler, Dene

Lo, Huang-Mu

Christensen, Morten Lykkegaard

Robeson, Lloyd M.

Madsen, Henrik T.

Magda, Jules J.

Marquardt, Drew

Matsumoto, Michiaki

Matsuyama, Hideto

Molugu, Sudheer Kumar

$\mathrm{Na}$, Huimin

Nagarajan, Sureshbabu

Nagy, Endre

Nijmeijer, Arian

Nikles, Jacqueline A.

Orchard, Sandra

Parisi, Salvatore

Patsios, Sotiris I.

Peters, Judith

Piacentini, Emma

Pihlajamäki, Arto

Pouliot, Yves

Rajca, Mariola

Ribeiro, Clarisse
Romero-Vargas Castrillón, Santiago

Roy, Sagar

Ruiz-García, Alejandro

Sahadevan, Rajesh

Schirhagl, Romana

Schramm, Christian

Schroen, Karin

Schulze, Agnes

Schwank, Johannes

Selyanchyn, Roman

Sentana, Irene

Sheehan, Diarmuid

Shen, Hsin-Hui

Shrivastava, Abhishek

Siebenhofer, Matthäus

Silva, Liana C.

Srinivas, Keerthi

Szekely, Gyorgy

Tachikawa, Masashi

Tahir, Nawaz

Takemoto, Hiroyasu

Tizaoui, Chedly

Tobino, Tomohiro

Torrieri, Elena

Uchihashi, Takayuki

Van Griensven, Leo J.L.D

Vargiu, Attilio V.

Vaz, Deisi Altmajer

Wang, Haining

Wang, Tanyu

Wang, Tuo

Woody, Robert W.

Wu, Bing

Yabu, Hiroshi

Yang, Jen Ming

Yin, Jun

Yuan, Pao-chiang

Yuba, Eiji

Zarca, Gabriel

Zhang, Peng

Zhu, Shan

Zornoza, Beatriz

(C) 2017 by the author; licensee MDPI, Basel, Switzerland. This article is an open access article distributed under the terms and conditions of the Creative Commons Attribution (CC-BY) license (http://creativecommons.org/licenses/by/4.0/). 